Visualizing Objects, Places, and Spaces: A Digital Project Handbook

\title{
Budgets \& Funding for Temporal Projects
}

Beth Fischer, Hannah Jacobs, Kemba N'Namdi

Published on: Oct 14, 2019

DOI: $10.21428 / 51$ bee781.257c82fo

License: Creative Commons Attribution 4.0 International License (CC-BY 4.0). 


\section{Budget}

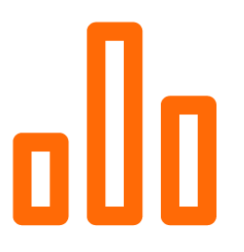

Many temporal projects have no direct upfront costs at all. The most common programs used for temporal projects are free and many are entirely open source, though some have paid options for hosting large amounts of content online or providing advanced services.

If a project requires customization, you may need to hire a web developer, often someone with expertise in javascript, or other programming skills. The cost will vary depending on the complexity of the customization and the skills required. You can keep costs for this down by starting with an open-source program that meets most of your needs (perhaps something like timelineJS) and making minimal additions. Changes relating to appearance -- size, colors, layout proportions, and similar features -- will typically be easier and cheaper than changes to core functionality like adding parallel timelines or complex paths. If you have more time than money, or if you have a strong interest in learning to edit javascript, there are a lot of free resources online for learning this type of coding and a lot of relevant javascript libraries that can provide code pieces that you can edit together so that you aren't starting from scratch.

If you are considering features that do not come in free versions, you will need to look at the difference between paying a higher one-time amount for custom coding and an ongoing subscription cost for a paid tier of a commercial timeline maker. The cost for paid versions is usually under $\$ 100 /$ year, so if your project will only be up for a semester or a few months, this is a cheaper and faster solution if it meets your needs. Make sure to take into account how to archive or preserve your timeline if you use a subscription service and allow it to expire!

You should also consider whether you will have costs associated with gathering, digitizing, or paying for publication rights for any of your source materials. Possible costs associated with digitizing content are covered in the description of budgets for archival projects.

The final cost you may encounter is simply for hosting your source materials or a website that displays your project. Many timeline platforms will host the timeline itself and allow you to embed it in your own website, but typically they require you to host images, custom maps, and other multimedia in your own server space. 
Unless a timeline project involves hundreds of files or uses lots of videos or $3 \mathrm{~d}$ models, you can probably host the necessary amount of material using the free level of services like Google Drive, Dropbox, or Box. The costs for hosting large files are addressed in more detail in the description of budgets for archival projects.

\section{Funding}

The impetus for temporal projects is often the desire to explain complicated relationships between events in a better way. For that reason, the best routes for external funding are often educational outreach or curriculum development grants rather than funding explicitly marked for digital projects. Even if you need to hire a freelance programmer, costs are likely to be in the hundreds or low thousands, so sometimes internal departmental budgets or institutional faculty development grants can be sufficient. If the project is essential to an ebook or other publication, you can ask administrators of publications grant programs whether this sort of material development is fundable.

If the temporal project requires an innovative solution, something that requires technological research rather than just the customization of code, you might look into digital humanities grants, but this is likely to be a very rare situation.

Continue Reading: Prototyping \& Wireframing Temporal Projects 